\title{
Long-term experience with GH replacement therapy: efficacy and safety
}

\author{
John P Monson
}

Department of Endocrinology, Division of General and Developmental Medicine, St Bartholomew's Hospital, West Smithfield, London ECIA $7 B E$, UK

(Correspondence should be addressed to J P Monson; Email: J.P.Monson@qmul.ac.uk)

\begin{abstract}
Demonstration of the long-term efficacy of GH replacement in GH-deficient adults has depended on a combination of single-centre studies and data from large multinational databases, which, by virtue of their size, are likely to detect rare adverse events and also permit analysis of mortality rates. The Pharmacia International Metabolic Surveillance (KIMS) study (a pharmacoepidemiological survey of the safety and efficacy of GH replacement in adults, sponsored by Pharmacia) is currently the largest database, with information on over 8000 patients from a total of 27 countries.

Abundant epidemiological evidence confirms that hypopituitarism is associated with premature mortality, with an increase in cardiovascular and cerebrovascular disease as a primary underlying cause. Central adiposity, hyperlipidaemia, insulin resistance, and diabetes mellitus are common in adults with hypopituitarism. GH replacement is associated with improvements in central fat mass and mean reductions in serum total and low-density lipoprotein cholesterol which may be additive to those achieved with hydroxymethylglutaryl-coenzyme A reductase inhibitors. These beneficial effects are maintained for at least 2 years after initiation of therapy, as are reductions in central adiposity, with similar benefits seen in men and women when the GH dose is titrated to achieve a serum IGF-I between the median and the upper end of the age-related reference range. Fasting plasma glucose and glycated haemoglobin increase, usually within the reference range, during prolonged GH replacement, but do not tend to rise further above baseline in subjects with pre-existing impaired glucose tolerance.

Bone remodelling increases during GH replacement therapy, but indices tend to return to baseline within 5 years of commencing treatment. Bone mineral density increases in men whereas, in women, improvement is limited to stabilisation of bone density. Data from the KIMS study demonstrate that prolonged GH replacement is associated with a reduction in the number of patients requiring assistance with daily living and a significant reduction in sick leave and hospital admissions.

GH replacement therapy improves psychological well-being, particularly in those patients with the greatest deficit prior to treatment, with improvement maintained beyond 6 months of therapy and sustained during long-term follow-up.

Data from the KIMS population show that there is no increase in the overall occurrence of de novo neoplasia or the rate of regrowth of primary pituitary tumours. There is an apparent increase in intracranial neoplasia, which may be an artefact of comparing a surveillance population with general population data. Unlike mortality in untreated hypopituitary GH-deficient patients, mortality in the KIMS study is currently similar to that predicted for the normal population.
\end{abstract}

European Journal of Endocrinology 148 S9-S14

\section{Introduction}

Growth hormone (GH)-deficient adults generally experience a constellation of signs and symptoms including fatigue and abnormal body composition, and metabolic changes that increase the risk for cardiovascular disease and bone fracture and may lead to greater overall mortality (1-6). The consequences of GH deficiency (GHD) also result in elevated direct and indirect costs. One study noted a significant increase in in-patient care and use of disability pension and sick leave in patients with GHD compared with the general population (7). Fortunately, GH replacement therapy significantly improves the metabolic profile of these patients and enhances quality of life (1). However, despite these initial improvements noted with $\mathrm{GH}$ replacement therapy, data on the long-term efficacy and safety of GH are limited.

To address the lack of long-term evidence, an international surveillance programme was initiated in 1994 to collect data on patients who have GHD and are receiving GH replacement therapy. The objectives of this study, entitled The Pharmacia International Metabolic Surveillance (KIMS) study, was to determine the clinical characteristics of adult patients with GHD, the long-term safety and efficacy of GH replacement therapy in adult patients with GHD, and the effects of GH replacement on mortality. When this paper was written, the ongoing surveillance programme included more than 8000 adult patients ranging in age from 
18 to 82 years. This paper will provide an overview of GHD and present the long-term data currently available from the KIMS study and single centre studies on GH replacement therapy in adults.

\section{Overview of GHD}

The majority of patients included in the KIMS database have pituitary adenomas (Fig. 1), which are the cause of GHD in approximately $59 \%$ of patients aged 18-65 years and $84 \%$ of patients aged $65-82$ years (8). Craniopharyngioma is the second most frequent cause of GHD in both age groups, with a slightly higher incidence in younger patients (i.e. $12 \%$ vs $4 \%$ in patients aged 65 and older). Other causes of GHD include surgery or radiotherapy for the treatment of other non-pituitary pathologies, and cranial irradiation $(9,10)$. GHD should also be suspected in patients with a history of infiltrative diseases such as sarcoidosis, histiocytosis, or lymphocytic hypophysitis, and head trauma or vascular injury.

Typical signs of GHD include increased fat mass, particularly central adiposity, decreased lean body mass, and reduced exercise capacity and muscle strength, particularly in active individuals. Thin, dry skin with cool extremities is also noted in GHD. These patients may also exhibit an impaired sense of psychological well-being, depressed mood, social anxiety, and fatigue $(11,12)$. The diagnosis of severe GHD is based on a stimulated $\mathrm{GH}$ concentration less than $3 \mu \mathrm{g} / \mathrm{l}$, on an insulin tolerance test, arginine stimulation or glucagon test $(2,10)$. Additionally, most patients with GHD exhibit a reduced serum insulin-like growth factor-I (IGF-I) concentration. Increased low-density lipoprotein (LDL)-cholesterol concentration, and reduced bone mineral density are additional common features (11).

Adults with GHD are also at risk for cardiovascular disease. Premature atherosclerosis has been noted in

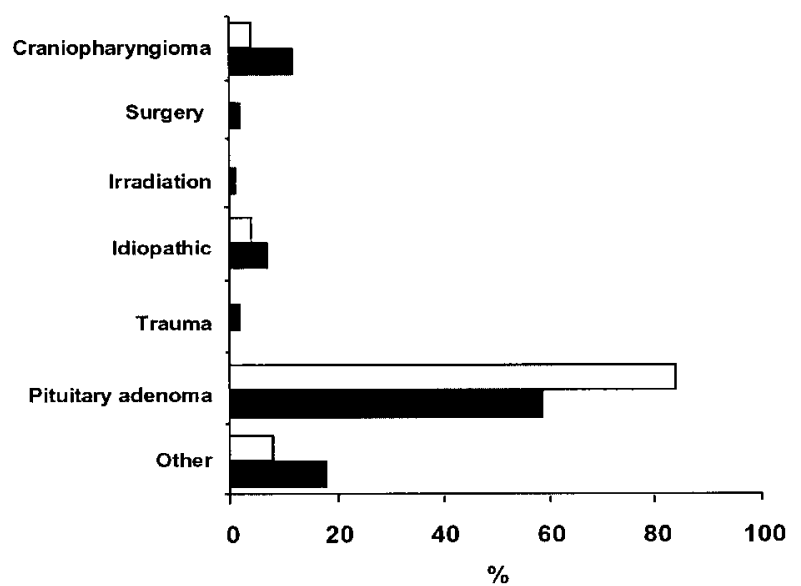

Figure 1 Aetiology of GH deficiency in patients of more than 65 years (open bars) and less than 65 years (solid bars) (8). many patients with untreated GHD. GHD is associated with increased very low-density lipoprotein (VLDL) secretion and decreased clearance of VLDL and LDL. Subsequent reductions in high-density lipoprotein (HDL) also occur. Increased atherogenesis is manifest by increased intima-medial thickening, intimal plaque formation, and decreased arterial compliance $(11,12)$. All of these factors predict accelerated atherosclerosis, leading to complications of cardiovascular disease (e.g. myocardial infarction, heart failure). Reduced left ventricular mass, impaired cardiac systolic function, and reduced cardiac output are often found in patients with GHD $(13,14)$.

Baseline lipid data of the first 1034 patients with GHD enrolled in the KIMS study revealed that $39.2 \%$ of men and $41.8 \%$ of women had an LDL-cholesterol level greater than $4 \mathrm{mmol} / \mathrm{l}$ (1). A comparison of those findings with LDL concentrations from the general population found that significantly more patients with GHD have elevated LDL concentrations; only $14.8 \%$ of men and $16.6 \%$ of women in the general population have an LDL greater than $4 \mathrm{mmol} / \mathrm{l}(P<0.0005)$ (1). A similar trend was also seen with triglyceride measurements, as patients with GHD had significantly higher concentrations than those of the general population $(P<0.05)(1)$.

Alterations in bone metabolism are another significant finding in patients with GHD. Reductions in bone remodelling, particularly reduced osteoblastic activity, are thought to occur, resulting in osteopenia and an increased rate of fracture (15). The degree of osteopenia appears to be directly related to the severity of GHD (16). The exact impact of GHD on bone metabolism is unclear because many factors can confound this relationship. Most patients with GHD have other pituitary hormone deficiencies and are receiving replacement corticosteroids, which can negatively impact bone mineral density. Additionally, hypogonadism resulting in decreased concentrations of oestrogen and testosterone can reduce bone mass. Despite these confounding factors, an absence of GH appears to play a major role in the development of osteopenia and fracture because patients with isolated GHD appear to have similar changes in bone mineral density to those with multiple hormone deficiencies (17). Significant alterations in bone metabolism were evident in the KIMS study as patients with hypopituitarism or isolated GHD had low spinal bone mineral density measurements (15). In the 2084 patients evaluated, 578 fractures were detected, with radial fractures being the most frequently documented fracture type. A comparison of the GH-naïve KIMS population with the general population showed a 2.66-fold increase in fracture risk in patients more than 60 years of age. This finding was statistically significant in men $(P<0.05)(15)$.

Patients with GHD also complain of reductions in quality of life and psychological well-being. Depression, anxiety, and decreased energy are associated with GHD. 
Conventional treatment does not seem to alleviate these symptoms, suggesting that GH plays an important role in quality of life $(11,12)$. A quality of life questionnaire, called the Quality of Life-Assessment of GH Deficiency in Adults (QoL-AGHDA), was specifically developed to assess the impact of GHD on quality of life (18). In the KIMS population, the average score on the QoLAGHDA questionnaire was $9.0 \pm 6.4$ (range: $0=$ no problems to $25=$ severe impairment), suggesting a moderate degree of impairment in quality of life (19). These psychological complaints appear to correlate with reduced socioeconomic performance (19).

Compared with the general population, mortality is approximately doubled in patients with hypopituitarism including GHD (Fig. 2). The increased mortality appears to result from an increase in cardiovascular and cerebrovascular disease $(3,5)$. A retrospective study of 333 consecutive patients with hypopituitarism including GHD found an increased risk of death in patients with hypopituitary disease compared with the expected mortality in the general population $(P<0.001)$. The majority of patients with pituitary dysfunction died from a vascular disorder (e.g. myocardial infarction, cardiac failure, stroke) (3). Another study also noted a significant increase in vascular-related mortality in patients with hypopituitarism (5). This investigation found cerebrovascular disease, specifically, to be the primary cause of death. Women were noted to exhibit a higher mortality due to cerebrovascular disease compared with men as the SMR in women was $4.91(95 \%$ confidence interval (CI), 2.62-8.40) vs 2.64 in men (95\% CI, 1.44-4.42). Additionally, patients diagnosed with hypopituitarism before the age of 55 were at greater risk for cerebrovascular death (SMR of 6.67 (95\% CI, 3.38-12.1) vs 2.52 (95\% CI, 1.47-4.26) in patients diagnosed at 55 years or older). Although accelerated atherosclerosis and cardiovascular disease are thought to be the underlying causes of the shortened life expectancy of patients with GHD, other

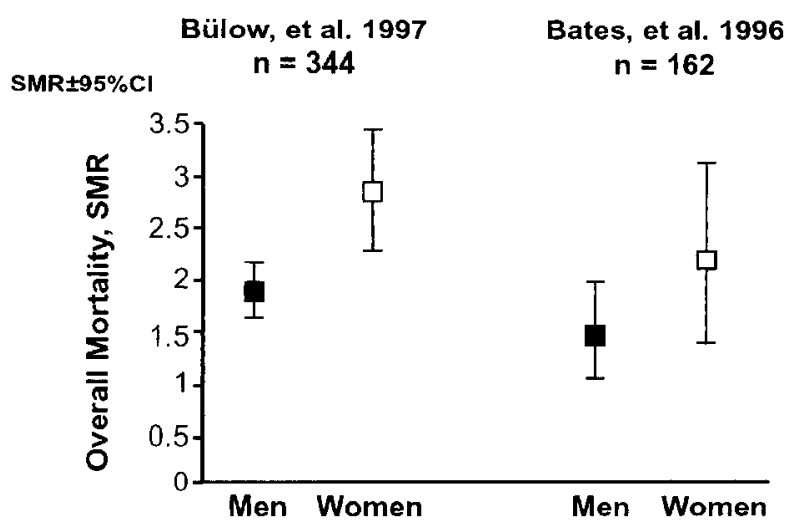

Figure 2 Mortality in men and women with hypopituitarism (Bülow et al. (5) and Bates et al. (4)). SMR, standardised mortality ratio. factors are also probably involved (e.g. underlying pituitary diagnosis, especially craniopharyngioma, and untreated gonadal steroid deficiency) (6).

\section{GH replacement therapy in GHD}

Short-term use of GH in patients with GHD improves body composition, reduces cholesterol concentration, elevates mood, and promotes a sense of well-being (11). Based on the data from the KIMS study, it appears that long-term administration of GH maintains these beneficial effects. In a single-centre study, compared with baseline measurements, 50 middle-aged patients with GHD achieved a significant reduction in their waist: hip ratio within 6 months of starting GH replacement therapy by dose titration $(P \leq 0.001)$, and the improvement was sustained at 1 year (20). Another long-term international surveillance investigation, the Hypopituitary Control and Complication Study, also showed long-term benefit in body composition with GH replacement (21). After 3 years of treatment, lean body mass was significantly increased $(P<0.001$ vs baseline) and percentage of body fat decreased in patients with adult-onset GHD. Additionally, a significant reduction in waist:hip ratio was sustained long term in men with adult-onset disease ( $P=0.017$ vs baseline).

Marked, long-term reductions in total cholesterol were also documented in several studies after the commencement of $\mathrm{GH}$ replacement therapy. In one study, serum total cholesterol concentration was significantly decreased in men $(-0.36 \pm 0.96 \mathrm{mmol} / \mathrm{l} ; P=$ 0.004 vs baseline) and women $(-0.32 \pm 1.00 \mathrm{mmol} / \mathrm{l}$; $P=0.045$ vs baseline) who were receiving $\mathrm{GH}$ but not lipid-lowering medications (21). Serum HDL-cholesterol concentration was increased by approximately $0.1 \mathrm{mmol} / \mathrm{l}$ from baseline in both men and women $(P=0.022$ and $P=0.026$ respectively vs baseline), and serum LDL-cholesterol concentration was significantly decreased from baseline in all patients with adult-onset disease. In a further single-centre study, during the initial 6 months of treatment, LDL-cholesterol concentrations decreased from 3.85 $0.19 \mathrm{mmol} / \mathrm{l}$ at baseline to $3.42 \pm 0.26 \mathrm{mmol} / \mathrm{l}$ with treatment $(P<0.05)$. These reductions were sustained for 18 months (22). Triglyceride concentrations were unaffected by GH treatment throughout the study period, and significant improvements from baseline in serum HDL-cholesterol concentrations were noted after 18 months of $\mathrm{GH}$ replacement therapy $(1.24 \pm$ 0.11 vs $1.08 \pm 0.1$ at baseline, $P<0.05)$. The greatest reductions in cholesterol concentrations were seen in those patients with the highest total cholesterol concentrations at baseline, and the reductions occurred regardless of whether patients were already taking lipid-lowering therapy at study entry. A separate analysis of 10 patients receiving hydroxymethylglutaryl-coenzyme A (HMG-CoA) reductase inhibitor 
therapy for at least 6 months before GH initiation found a significant reduction in total cholesterol after 6 months of treatment $(5.62 \pm 0.22 \mathrm{mmol} / \mathrm{l}$ at baseline vs $5.03 \pm 0.28 \mathrm{mmol} / \mathrm{l} ; P<0.05$ ) (Fig. 3). The $10 \%$ reduction in LDL-cholesterol concentration noted in the study (22) would predict a $20-30 \%$ reduction in cardiovascular risk (23).

In a small open-label study of 15 patients with GHD, the long-term effects of $\mathrm{GH}$ replacement on bone metabolism and bone mineral density were evaluated (24). A significant increase in osteoblastic activity as measured by serum bone-specific alkaline phosphatase concentration was noted during 6 months of therapy, but returned to baseline by study end at 5 years. These findings suggest an early effect of $\mathrm{GH}$ on bone remodelling. After a median of 58 months (range 44-72 months), GH replacement therapy was associated with a significant increase in lumbar spine and femoral neck bone mineral density in men $(P=0.01$ and $P=0.02$ respectively), although bone mineral density remained stable in women (Fig. 4) (24). Using a larger patient population, a second study also evaluated the long-term effects of GH replacement on bone mass (25). Over a 5-year period, 118 patients with adult-onset GHD received decreasing doses of $\mathrm{GH}$. Total bone mineral content (BMC) was increased by $2 \%$ during the treatment period, and the BMC of the lumbar and femur neck also improved by 7 and $6 \%$ respectively $(P<0.001$ for lumbar BMC vs baseline and $P<0.01$ for femur neck BMC vs baseline). Additionally, bone mineral density in the lumbar spine and femur neck were normalised (i.e. Z-score $\geq 0$ ) with long-term GH therapy. These effects were noted after 2 years of treatment and were sustained throughout the study period (25).

Quality of life improvements also occur acutely with GH replacement therapy and appear to be maintained long term. In the KIMS population, decreases in QoLAGHDA scores of three to eight points were noted during the first 12 months of GH replacement therapy, indicating a marked improvement in quality of life $(P<0.001$ vs baseline $)(19)$. To access a patient's

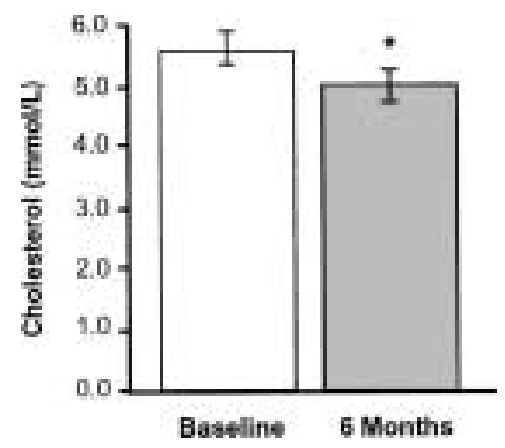

Figure 3 Serum cholesterol after 6 months of GH therapy in ten patients receiving an HMG-CoA reductase inhibitor (22). Values are means \pm S.E.M. ${ }^{*} P<0.005$.
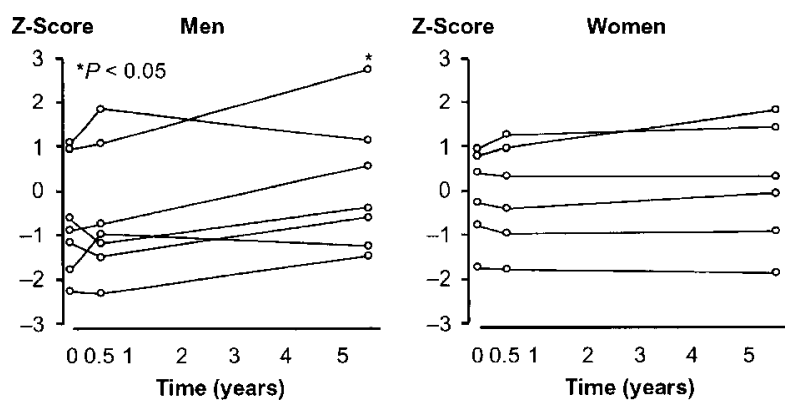

Figure 4 Change in lumbar spine bone mineral density Z-score with GH therapy. (Reprinted, with permission, from (24).)

personal situation (e.g. marital status, education, employment), all participants in the KIMS study completed a patient life situation questionnaire at baseline and annually thereafter. Data collected on 304 patients indicated that, after 12 months of GH replacement therapy, both men and women participated in significantly more leisure activities compared with baseline $(P<0.001)$. This increase in activity was mirrored by a marked improvement in patient satisfaction with their physical condition (Fig. 5). Requirements for assistance with daily activities also diminished significantly $(P<0.05)$ for both men and women. Furthermore, the number of hospital days and physician visits decreased during this period $(P<0.05$ vs baseline for all measurements) and the number of days of sick leave decreased from a mean of $9.5 \pm 30.4$ days to $3.8 \pm$ 13.2 days $(P<0.05)$ (19). These improvements in quality of life, physical activity, patient satisfaction, and consumption of health care resources remained stable through 24 months of treatment (19).

\section{Adverse effects of GH replacement therapy}

GH replacement therapy appears relatively safe in patients with GHD. In an analysis from the KIMS database including 1034 patients for a total of 818 patient years, the incidence of adverse events was 1.08 per patient year (1). The most common adverse effects with GH therapy included respiratory tract infection, oedema and stiffness, arthralgia, myalgia, and pain in the extremities, headache or migraine, diarrhoea, and hypertension. In this analysis of the KIMS data, only $3.5 \%$ of patients discontinued therapy (1). Adverse effects have been reduced by the use of dose titration against serum IGF-I in place of weight-based dosing regimens (16).

One concern with GH replacement therapy is its impact on carbohydrate metabolism. Reports of diabetes mellitus and increased fasting blood glucose concentrations have been noted following initiation of GH therapy in children (10). A study in eight adults 


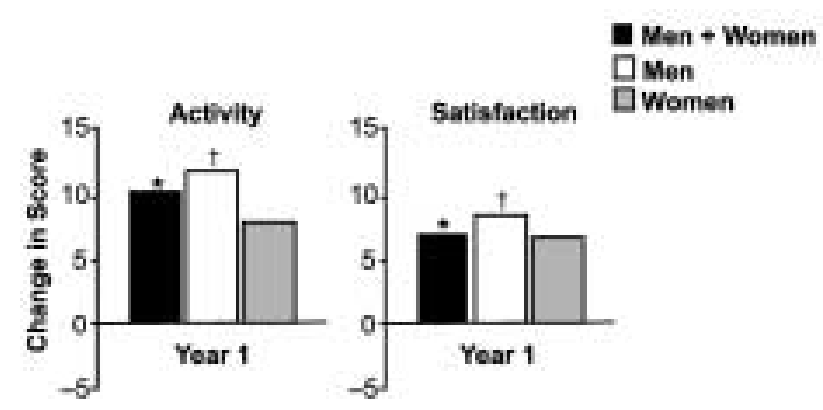

Fig. 5 Improvements in physical activity and patient satisfaction after 1 year of GH therapy (19). ${ }^{\star} P<0.001$ vs baseline; $\dagger P<0.05$ vs women.

with GHD evaluated the effects of GH replacement on insulin sensitivity (26). In this investigation, serum fasting blood glucose and cholesterol concentrations, blood pressure, and body weight were unchanged after 18 months of GH replacement. Glycated haemoglobin $\left(\mathrm{HbA}_{1 \mathrm{c}}\right)$ and triglyceride levels were increased after 12 months of therapy but normalised with continued treatment. In contrast to other studies (which have demonstrated a reduction in insulin sensitivity at baseline decreasing further with $\mathrm{GH}$ replacement initially but returning to baseline at around 12 months), insulin sensitivity was documented to be higher in GHdeficient patients and to normalise after 18 months of $\mathrm{GH}$ replacement. This decrease in insulin sensitivity corresponded with an increase in basal and total insulin secretion during the study period. The investigators concluded that patients with a family history of diabetes mellitus or compromised $\beta$-cell function should be closely monitored for a worsening of glycaemic control during GH therapy (26).

In a further study in 90 patients who completed 2 years of treatment, data on fasting plasma glucose and $\mathrm{HbA}_{1 \mathrm{c}}$ concentrations were reported (22). A slight, but statistically significant, increase in plasma glucose and serum $\mathrm{HbA}_{1 \mathrm{c}}$ concentrations was noted within 6 months of starting GH replacement therapy. Fasting plasma glucose concentration increased from 4.72 $0.06 \mathrm{mmol} / \mathrm{l}$ at baseline to $5.15 \pm 0.07 \mathrm{mmol} / \mathrm{l}$ after 6 months $(P<0.001)$, and $\mathrm{HbA}_{1 \mathrm{c}}$ values rose from $4.9 \pm$ 0.05 to $5.07 \pm 0.06(P<0.001)$. These elevations were sustained for 2 years, but the values remained within the normal reference range. A subanalysis of patients based on pretreatment $\mathrm{HbA}_{1 \mathrm{c}}$ values showed that $\mathrm{HbA}_{1 \mathrm{c}}$ increased slightly in patients without evidence of glucose intolerance at baseline while most patients with slightly elevated pretreatment $\mathrm{HbA}_{1 \mathrm{c}}$ levels demonstrated a reduction or no change in $\mathrm{HbA}_{1 \mathrm{c}}$. Changes in carbohydrate metabolism were unrelated to the dose of $\mathrm{GH}$, but the increase in $\mathrm{HbA}_{1 \mathrm{c}}$ correlated with a higher baseline body mass index (BMI) (22).

Pituitary tumours are the most common cause of GHD. The possible effects of GH replacement therapy on tumour growth have been a cause of concern.
One investigation evaluated the effects of GH therapy on tumour growth in 100 adult patients who developed hypopituitarism following treatment for a pituitary or peripituitary tumour (27). Ninety-one percent of these patients had received external radiotherapy as part of their tumour treatment. Only one patient experienced an expansion of residual intrasellar tumour after 6 months of treatment. However, the patient continued $\mathrm{GH}$ therapy, and no further increases were noted after 1 year of treatment. No other patients experienced an increase in pituitary or peripituitary tissue (27). In the KIMS study, the overall incidence of recurrent pituitary tumours was not significantly different from that predicted for a treated pituitary tumour population (1). Although the incidence of de novo neoplasia in the KIMS study is not significantly different from that of the general population, a higher rate of intracranial tumours has been noted. This may be an incidental finding in a population under surveillance but may also reflect the fact that patients with pituitary tumours have a higher incidence of intracranial neoplasia regardless of therapy.

GH replacement therapy appears to be associated with a similar mortality to that of background populations. Excluding patients with a history of acromegaly or Cushing's disease, an analysis of 1903 patients from the KIMS database identified only 11 deaths (28). A comparison of the KIMS mortality with that of a Swedish population of hypopituitary patients treated with conventional therapy identified a mortality risk ratio of 3.25 for conventional treatment vs GH replacement therapy $(95 \%$ CI, 1.64-8.07). Thus, these results suggest that $\mathrm{GH}$ replacement therapy may have a favourable impact on the previously demonstrated increased mortality of hypopituitarism (28).

\section{Conclusions}

The adult GHD syndrome is now well recognised. Patients with this syndrome exhibit debilitating signs and symptoms, affecting the cardiovascular system, bone metabolism, and quality of life. GH replacement therapy is associated with several positive benefits in these patients. Clinical trials have documented improvements in body composition, lipid profile, and bone mineral density following GH treatment. Additionally, $\mathrm{GH}$ replacement therapy appears to produce important physiological benefits, which have positive impact on psychological well-being. The net effect of GH replacement on cardiovascular risk is likely to be determined by the net effect of the beneficial effects of treatment on central adiposity and lipid metabolism on the one hand with the adverse effect on insulin sensitivity demonstrated in some patients, especially those with high BMI.

GH replacement therapy appears to be well tolerated, and the discontinuation rate is low. Mortality to date among recipients of GH replacement therapy is similar 
to that of the general population, which suggests that treatment may reduce mortality in the at-risk population with GHD. The occurrence of secondary cancer does not appear to be increased, but longer duration of follow-up is required for confirmation.

\section{References}

1 Abs R, Bengtsson BA, Hernberg-Stahl E, Monson JP, Tauber JP, Wilton P et al. GH replacement in 1034 growth hormone deficient hypopituitary adults: demographic and clinical characteristics, dosing and safety. Clinical Endocrinology $199950703-713$.

2 Lamberts SW, De Herder WW \& Van Der Lely AJ. Pituitary insufficiency. Lancet 1998352 127-134.

3 Rosen T \& Bengtsson BA. Premature mortality due to cardiovascular disease in hypopituitarism. Lancet 1990336 285-288.

4 Bates AS, Van't Hoff W, Jones PJ \& Clayton RN. The effect of hypopituitarism on life expectancy. Journal of Clinical Endocrinology and Metabolism 199681 1169-1172.

5 Bülow B, Hagmar L, Mikoczy Z, Nordstrom CH \& Erfurth EM. Increased cerebrovascular mortality in patients with hypopituitarism. Clinical Endocrinology 199746 75-81.

6 Tomlinson JW, Holden N, Hills RK, Wheatley K, Clayton RN, Bates AS et al. Association between premature mortality and hypopituitarism. West Midlands Prospective Hypopituitary Study Group. Lancet 2001357 425-431.

7 Ehrnborg C, Hakkaart-Van Roijen L, Jonsson B, Rutten FF, Bengtsson BA \& Rosen T. Cost of illness in adult patients with hypopituitarism. PharmacoEconomics 200017 621-628.

8 Monson JP, Abs R, Bengtsson BA, Bennmarker H, FeldtRasmussen U, Hernberg-Stahl E et al. Growth hormone deficiency and replacement in elderly hypopituitary adults. KIMS Study Group and the KIMS International Board. Pharmacia and Upjohn International Metabolic Database. Clinical Endocrinology $200053281-289$

9 American Association of Clinical Endocrinologists, AACE clinical practice guidelines for growth hormone use in adults and children. Endocrine Practice 19984 165-173.

10 Thorner MO, Bengtsson B- $\AA$, Ho KKY, Albertsson-Wikland KM, Christiansen JS, Faglia G et al. Consensus guidelines for the diagnosis and treatment of adults with growth hormone deficiency: summary statement of the Growth Hormone Research Society Workshop on Adult Growth Hormone Deficiency. Journal of Clinical Endocrinology and Metabolism 199883 379-381.

11 Carroll PV, Christ ER \& Sonksen PH. Growth hormone replacement in adults with growth hormone deficiency: assessment of current knowledge. Trends in Endocrinology and Metabolism 2000 $11231-238$.

12 Cuneo RC, Salomon F, McGauley GA \& Sonksen PH. The growth hormone deficiency syndrome in adults. Clinical Endocrinology $199237387-397$

13 Cuocolo A, Nicolai E, Colao A, Longobardi S, Cardei S, Fazio S et al. Improved left ventricular function after growth hormone replacement in patients with hypopituitarism: assessment with radionuclide angiography. European Journal of Nuclear Medicine 1996 23 390-394.

14 Colao A, Cuocolo A, Di Somma C, Cerbone G, Della Morte AM, Nicolai E et al. Impaired cardiac performance in elderly patients with growth hormone deficiency. Journal of Clinical Endocrinology and Metabolism $1999 \mathbf{8 4} 3950-3955$.

15 Wuster C, Abs R, Bengtsson BA, Bennmarker H, FeldtRasmussen U, Hernberg-Stahl E et al. The influence of growth hormone deficiency, growth hormone replacement therapy, and other aspects of hypopituitarism on fracture rate and bone mineral density. Journal of Bone and Mineral Research 200116 398-405.

16 Drake WM. Howell SJ. Monson JP \& Shalet SM. Optimizing GH therapy in adults and children. Endocrine Reviews 200122 $425-450$.

17 Kaufman JM, Taelman P, Vermeulen A \& Vandeweghe M. Bone mineral status in growth hormone-deficient males with isolated and multiple pituitary deficiencies of childhood onset. Journal of Clinical Endocrinology and Metabolism 1992 74 118-123.

18 McKenna SP, Doward LC, Alonso J, Kohlmann T, Niero M, Prieto L et al. The QoL-AGHDA: an instrument for the assessment of quality of life in adults with growth hormone deficiency. Quality of Life Research 19998 373-383.

19 Hernberg-Stahl E, Luger A, Abs R, Bengtsson BA, FeldtRasmussen U, Wilton P et al. Healthcare consumption decreases in parallel with improvements in quality of life during GH replacement in hypopituitary adults with GH deficiency. Journal of Clinical Endocrinology and Metabolism 200186 5277-5281.

20 Drake WM, Coyte D, Camacho-Hubner C, Jivanji NM, Kaltsas G, Wood DF et al. Optimizing growth hormone replacement therapy by dose titration in hypopituitary adults. Journal of Clinical Endocrinology and Metabolism $1998 \mathbf{8 3} 3913-3919$.

21 Attanasio AF, Bates PC, Ho KK, Webb SM, Ross RJ. Strasburger CJ et al. Human growth hormone replacement in adult hypopituitary patients: long-term effects on body composition and lipid status 3-year results from the HypoCCS Database. Journal of Clinical Endocrinology and Metabolism 200287 1600-1606.

22 Florakis D, Hung V, Kaltsas G, Coyte D, Jenkins PJ, Chew SL et al. Sustained reduction in circulating cholesterol in adult hypopituitary patients given low dose titrated growth hormone replacement therapy: a two year study. Clinical Endocrinology 200053 453-459.

23 Law MR, Wald NJ, Wu T, Hackshaw A \& Bailey A. Systematic underestimation of association between serum cholesterol concentration and ischaemic heart disease in observational studies: data from the BUPA study. British Medical Journal $1994 \mathbf{3 0 8}$ 363-366.

24 Drake WM, Rodriguez-Arnao J, Weaver JU, James IT, Coyte D, Spector TD et al. The influence of gender on the short- and long-term effects of growth hormone replacement on bone metabolism and bone mineral density in hypopituitary adults: a 5-year study. Clinical Endocrinology 200154 525-532.

25 Gotherstrom G, Svensson J, Koranyi J, Alpsten M, Bosaeus I, Bengtsson B et al. A prospective study of 5 years of GH replacement therapy in GH-deficient adults: sustained effects on body composition, bone mass, and metabolic indices. Journal of Clinical Endocrinology and Metabolism 200186 4657-4665.

26 Riedl M. Ludvik B, Pacini G. Clodi M, Kotzmann H, Wagner O et al. The increased insulin sensitivity in growth hormone-deficient adults is reduced by growth hormone replacement therapy. European Journal of Clinical Investigation 200030 771-778.

27 Frajese G, Drake WM, Loureiro RA, Evanson J, Coyte D, Wood DF et al. Hypothalamo-pituitary surveillance imaging in hypopituitary patients receiving long-term GH replacement therapy. Journal of Clinical Endocrinology and Metabolism 200186 5172-5175.

28 Bengtsson BA, Koppeschaar HP, Abs R, Bennmarker H, HernbergStahl E, Westberg B et al. Growth hormone replacement therapy is not associated with any increase in mortality. KIMS Study Group. Journal of Clinical Endocrinology and Metabolism $1999 \mathbf{8 4}$ 4291-4292.

Received 6 December 2002

Accepted 20 December 2002 